\title{
Towards Utilizing Flow Label IPv6 in Implicit Source Routing for Dynamic Source Routing (DSR) in Wireless Ad Hoc Network
}

\author{
${ }^{1}$ Wai Yee Tai, ${ }^{2}$ Chong Eng Tan, ${ }^{3}$ Sei Ping Lau \\ Department of Computer Systems and Communication Technologies, \\ Faculty of Computer Science and Information Technology, Universiti Malaysia Sarawak, Malaysia \\ e-mail: ${ }^{1}$ whyee86@gmail.com, $\left\{{ }^{2}\right.$ cetan, ${ }^{3}$ splau $\} @$ fit.unimas.my
}

\begin{abstract}
As Internet Protocol version 4 (IPv4) addresses deplete, Internet Protocol version 6 (IPv6) is introduced to alleviate the depletion with introduction of much needed functionality in security and so much more. Quality of Service $(\mathrm{QoS})$ has been one of the aspects taken into consideration for improving network performance such as Packet Delivery Fraction (PDF), average End-to-end Delay and throughput. However in IPv4 network transmission, QoS is not given much attention as all transmission is treated equally with their "best-of-effort" services. Hence, in wireless ad hoc networks where unpredictable changes in topologies often disrupt on-going transmission. It will affect network performances with only the "best-of-effort" basis especially on real-time applications that require good network metrics. Therefore, in this paper we show how IPv6 can play its part in improving wireless ad hoc networks with QoS in Dynamic Source Routing (DSR) routing protocol.
\end{abstract}

Keyword: IPv4, IPv6, QoS, Dynamic Source Routing, Wireless ad hoc network

\section{INTRODUCTION}

IPv4 was described in RFC 791 since 1981 is a connectionless protocol that works on best of effort delivery model. Best effort described as a method that does not guarantee the delivery nor make certain of their sequences. Moreover, it does not avoid duplications of delivery. In the early 1990s, IPv4 encountered IP address exhaustion and in order to alleviate the encountered problem, IPv6 is standardized in year 1998 [1]. IPv6 promises a larger address space to accommodate the exponential demands of IP addresses as well as improves the whole transmission in IPv6 networks. IPv6 is revamped for better efficiency in routers processing, added functionality for address configurations, multicasting ability and so much more. The transition from IPv4 to IPv6 are taking place and as migration takes place there will be no exception for wireless ad hoc network to stay unchanged.

Wireless ad hoc network comprises of nodes that mutually agreed to forward data on behalf of source node to their respective destinations. Routing in ad hoc network allows impromptu connections to be established among devices without any centralized management structure and therefore, routing protocol plays an important role. Routing protocol is used in determining the route for transmissions as well as seeking alternative path for any disrupted transmission. Routing protocols are generally divided into two categories which are On-Demand (Reactive) protocols and Table-Driven (Proactive) protocols. On-Demand routing protocols are reactive protocols in which the node will only search for potential routes when transmission is contrived whereas Table-Driven routing protocols are proactive protocols where nodes within will periodically exchange information to their neighboring nodes in attempts to get latest updates in order to have routes ready for transmission. Prominent routing protocols that are categorized as On-Demand protocols are DSR [2] and AODV [3] and while Table-Driven routing protocol that is well-known is DSDV [4].

DSR is one of the on-demand routing protocol that uses source routing concept which allows originator of transmissions to make routing decision. It is based on the source route that explicitly lists the route for the intermediate nodes in forwarding the packet [5]. This provides simplicity, correctness and flexibility to all nodes as the packet is forwarded to the next address as indicated in the source route packet's header. As IPv6 has a longer packet header in comparison to IPv4, it costs higher overhead in routing processes. However, with implicit source routing concept, packet processing is reduced. Besides, IPv6 packet header has lesser fields than IPv4 packet header in comparison. Implicit Source Routing [6] is introduced to reduce the routing packet overhead while preserving the on-demand ad hoc routing advantages.

In this paper, Section II briefly discussed IPv6 structure that is proposed to succeed the predecessor and the overview of DSR routing protocol with Implicit Source Routing. Section III shows different utilizations of IPv6 Flow Label in attempts to improve the network performance. Section IV proposes a new way of improving the DSR wireless ad hoc network performance and concludes this paper in Section VI. 